\section{Ogunlesi TA}

DOI:http://dx.doi.org/10.4314/njp.v40i1.2

Accepted: 29th May 2012

Ogunlesi, TA ( $)$

Department of Paediatrics, Olabisi Onabanjo University Teaching Hospital, Sagamu

P. O. Box 652, Sagamu-121001

Ogun State. Nigeria.

Email: tinuade_ogunlesi@yahoo.co.uk

\title{
Diagnosis and treatment of bacterial meningitis in the newborn \\ Diagnosis and treatment of bacterial
}

\begin{abstract}
Background: Bacterial meningitis in the newborn is globally renowned for high mortality. The associated morbidities also include audiologic, motor, visual and mental deficits.

Objective: To highlight the peculiarities in the current diagnostic and management strategies in newborn meningitis.

Methods: Relevant literature on the subject published only in English language or translated to English language was searched manually and electronically. The Medline, PUBMED and HINARI were searched for the period between 1966 and 2012. The following key words were used during the search: newborn/neonatal, bacterial/ pyogenic meningitis, central nervous system infections, antibiotics, dexamethasone and fluid restriction.

Results: The pattern of bacterial aetiology and mortality differ between the developed and developing world. The usefulness of bacte-
\end{abstract}

riologic culture in the diagnosis of meningitis can be improved with serologic method like polymerase chain reaction. Widespread resistance of pathogens may be threatening the use of penicillins and gentamicin for empirical treatment of newborn meningitis. No sufficient evidence presently supports the current practices of fluid restriction, prolonged duration of antibiotic treatment and non-use of adjuvant steroid therapies in the newborn. Conclusion: Efforts to reduce the incidence of newborn meningitis cannot be separated from the prevention of newborn sepsis generally. In addition, more controlled trials are required in the developing world with respect to the various aspects of management of newborn meningitis, particularly fluid management and the use of adjuvant steroids.

Keywords: Antibiotics, dexamethasone, meningitis, newborn, lumbar puncture.

\section{Introduction}

Meningitis is an extensive inflammatory condition of the leptomeninges. Although, it is generally described as uncommon, meningitis occurs commonly in the neonatal period due to the increased susceptibility of newborn babies to severe infections. 1 Neonatal meningitis is associated with significant mortality and severe morbidities.

The overall incidence of meningitis in England and Wales has not changed remarkably from the known 0.2 - 0.4 cases/1000 live births over the last three decades.

${ }^{2,3}$ These are similar to data obtained from other parts of the developed world. Although, consistent incidence rates are not available in most parts of the developing world, the few available ones are higher compared to the developed world. For instance, in Nigeria, the incidence of neonatal meningitis in the middle belt was reported to be 1.9/1000 live births four and 6.5/ 1000 live births in north-eastern Nigeria. ${ }^{5}$ Similarly, the incidence rate reported in the Panama in the mid-nineties was 3.5 cases/1000 live births. ${ }^{6}$

While the incidence has not changed remarkably, there has been a significant decrease in the mortality associated with meningitis over the last two decades due to improved antibiotic therapy and supportive care in most parts of the developed and industrialized world. On the contrary, morbidity has not changed significantly over this same period, even, in the developed world. The persistently high morbidity rate among the survivors of neonatal meningitis remains a major clinical issue and the need to minimize these morbidities is a challenge. ${ }^{2,3}$

Although, mortality rates in newborn meningitis vary by 
region, the known rates in the various regions of the world are not markedly different. For instance, mortality rates included 0.7-1.9/ 1000 live births in sub-Saharan Africa, 0.33-1.5 in the Middle East and North Africa and $0.4-2.8$ in the Americas and Caribbean. ${ }^{7}$ Mortality in the developed world had dropped from close to $50 \%$ to about $10 \%$ over the last decade whereas morbidity remains high at 15 to $60 \%$ among the survivors. ${ }^{8}$

Although, under-reporting is a challenge in the developing world, mortality associated with neonatal meningitis also varies between $30 \%$ and $60 \%$ while morbidity figures vary greatly. Five to seven a high proportion of the survivors in neonatal meningitis develop chronic handicapping conditions with serious medical and psychosocial implications such as cerebral palsy, mental retardation, seizure disorder, hemiplegia, deafness and blindness. ${ }^{5,9}$

Although, bacterial meningitis is an important cause of newborn death globally, the burden of neonatal sepsis and meningitis is most pronounced in the resource-poor parts of the world where the disease constitutes a significant proportion of neonatal admission and deaths. ${ }^{10,11}$

In spite of the similarity in the pathology and pathogenesis of meningitis in childhood, there are peculiarities with regard to the diagnosis and management of newborn bacterial meningitis. This review aims to highlight the challenges in the diagnosis and management of bacterial meningitis in the newborn in comparison with older children. Research issues which may possibly proffer solutions to the alarming high prevalence of neurologic sequelae and poor quality of life among the survivors of newborn bacterial meningitis will also be raised.

\section{Diagnosis}

Symptoms and signs

Bacterial meningitis can be extremely difficult to diagnose in the newborn because the symptoms and signs are often subtle and non-specific at the early stage of the disease. The clinical presentation of meningitis is indistinguishable from that of sepsis without meningitis. The early symptoms of meningitis include pyrexia, poor feeding, vomiting, lethargy or irritability. These clinical features may also characterize other newborn disorders such as hypoxic-ischaemic encephalopathy and metabolic derangements such as hypoglycaemia. Therefore, distinguishing between early meningitis and other neonatal illnesses mentioned above may be challenging and this is associated with the tendencies to frequently under -diagnose meningitis in the newborn.

With the progression of the disease, bulging fontanelle, shrill cry, apnoeas, seizures, opisthotonus and coma may occur. ${ }^{12}$ Although, these features are more specific features of meningitis and thus, facilitate diagnosis on clinical grounds, they occur quite late in the disease. Instructively, the classic signs of meningeal irritation in the older children, such as neck stiffness and positive Kernig sign or Brudzinski sign are often absent and unreliable among infants. Thus, the latter features should not form the core of clinical diagnosis of meningitis in the newborn. Due to the low sensitivity and specificity of the clinical predictors of this severe infectious disease, laboratory diagnosis is indispensable. ${ }^{12,13}$

\section{Bacteriology of newborn meningitis Microbiology}

In consonance with sepsis, the bacterial aetiology of neonatal meningitis differs between the developing and the developed world. In most developed countries, the leading pathogen in newborn meningitis is Group $B$ Streptococcus (GBS). Others include Escherichia coli, Listeria monocytogenes, other coliforms and lately, Streptococcus pneumoniae. ${ }^{1,14}$ Data obtained from England and Wales from 1985 to 1987 and from 1996 to 1997 showed very little change in the pattern of bacterial aetiology of neonatal meningitis over this period. GBS accounted for $39 \%$ to $48 \%$ of all cases. Others included Escherichia coli (18\% to 26\%), Streptococcus pneumoniae $(6 \%)$ and Listeria monocytogenes (5\% to $7 \%$ ). ${ }^{2,3}$ In a more recent French National survey, the leading pathogens in newborn meningitis included GBS (59\%), Escherichia coli (28\%), Gram-negative bacilli apart from Escherichia coli (4\%), other Streptococcus apart from GBS (4\%), Neisseria meningitidis (3\%) and Listeria monocytogenes $(1.5 \%) .{ }^{15}$

The pattern of bacteriological aetiology in neonatal meningitis differs in terms of the spectrum of organisms as well as the relative prevalence of individual organisms causing meningitis in most parts of the developing world. Escherichia coli was earlier reported as the leading aetiology in Nigeria ${ }^{16}$ and more recently in Kenya. ${ }^{17}$ Although E. coli was also commonly isolated in Nigeria, Staphylococcus aureus predominated in two other Nigerian reports. ${ }^{4,5}$ In addition, some reports of GBS predominance have also been made in parts of the developing world like Zimbabwe, Kenya and China. ${ }^{17-19}$ Interestingly, none of the studies from Nigeria ${ }^{4,5,16}$ reported GBS as aetiology of neonatal meningitis. Even most recent studies of newborn sepsis in Nigeria did not find GBS or reported very few cases. ${ }^{20-23}$

Listeria monocytogenes is also uncommonly encountered in this part of the world. ${ }^{17}$ On the other hand, Gram negative bacilli (with the exception of $E$. coli) have a global distribution but appear more common in the developing world, probably for reasons of poor hygiene.

The emergence of unusual organisms like Haemophilus influenza in Nigerian babies with meningitis ${ }^{5,24}$ poses new challenges in the treatment of newborn meningitis in this population. This organism is not usually taken into consideration when planning empirical antibiotic treatment for newborn meningitis in Nigeria. This may constitute technical delay in the commencement of appropriate antibiotic therapy in possible cases of Haemophilus influenzae infection among newborns.

The highlighted differences in the aetiology of meningitis between the developed and developing world may be 
explained in terms of population differences in the rate and pattern of colonization, genetic differences in immune response and differences in laboratory techniques for pathogen isolation and reporting. 7,25

\section{Lumbar puncture}

Positive bacteriologic culture of the cerebrospinal fluid (CSF) is the gold standard diagnostic procedure for bacterial meningitis. In the absence of bacteriologic culture facilities, Gram stain of CSF may also provide useful information upon which initial decisions on diagnosis and management can be based. Blood culture may also be positive in $40-60 \%$ of meningitis cases. ${ }^{17,19,26}$

The definitive method for diagnosis of meningitis lies in the chemistry and bacteriologic culture of the CSF since the clinical features of newborn meningitis are largely non-specific and blood culture may not always be as stated above. There have been controversies about the indications for lumbar puncture (LP) in newborn sepsis. Current concessions appear to be pivoted on the fact that, LP is only useful as part of the work-up for lateonset sepsis or when infants with early-onset sepsis have clinical features suggestive of meningitis. Available evidence is not in support of the use of LP in routine sepsis work-up for healthy babies with presumed sepsis. ${ }^{27}$ Unlike in the older child, raised intracranial pressure rarely occurs in newborn meningitis since the open sutures and fontanel allows expansion of the cranium when pressure is building up. Thus, the exclusion of elevated intracranial pressure prior to LP may not be a major concern in the newborn. Nevertheless, compromised cardio-respiratory functions are clear contraindications to performing LP in critically ill infants. However, a modified left lateral position with the hip flexed to $90^{\circ}$ but without flexion of the neck has been proposed as useful for LP in the presence of respiratory embarrassment. ${ }^{28}$ In all situations, when meningitis is suspected, inability to perform LP should not delay appropriate antibiotic treatment.

When it is available, the CSF specimen should be examined macroscopically for turbidity and microscopically for the presence of bacterial organisms. Instructively, turbidity of the CSF is determined by the number of pus cells present in the fluid though the number may vary widely. Therefore, when clinical suspicion is strong, microscopic examination should take precedence over macroscopic examination. Although, Gram stain may reveal the pathogens in more than three-quarter of cases, bacteriological culture is the gold standard for diagnosing meningitis in the absence of prior exposure to antibiotics. Abnormalities in cell count and biochemical parameters such as glucose and proteins are also important for making diagnosis. Due to physiological variations in the biochemical and cellular parameters of newborn $\mathrm{CSF}$, caution needs to be exercised in interpreting the parameters. The white cell counts in the newborn CSF has been shown to be age-specific. ${ }^{29}$

Thus, CSF cell count needs to be interpreted with caution in the diagnosis of neonatal bacterial meningitis.
Similar caution has been raised with respect to CSF glucose and protein concentrations among preterm babies with meningitis. ${ }^{30}$ Indeed, there have been reservations about the use of the CSF glucose and protein parameters in making the diagnosis of meningitis or assessing the success of treatment of meningitis because of the high variability in the values of the CSF parameters. ${ }^{26}$ In a large study, babies with meningitis had CSF cell count of 0 to $15,900 / \mathrm{mm}^{3}$, glucose ranging from 0 to $199 \mathrm{mg} / \mathrm{dl}$ and protein ranging from 41 to $1964 \mathrm{mg} / \mathrm{dl}$ compared to 0 to $90,000 / \mathrm{mm}^{3}, 0$ to $1089 \mathrm{mg} / \mathrm{dl}$ and 3 to $4122 \mathrm{mg} / \mathrm{dl}$ respectively among normal babies. ${ }^{26}$ Instructively, studies are yet to show to what extent this variability truly affects the diagnosis of meningitis in the newborn. In spite of the challenges posed by the variability highlighted above, most practitioners still stick to the traditional cut-off values of the various parameters: white cell count $>32 / \mathrm{mm}^{3}$ with more than $60 \%$ polymorphonuclear cells, proteins $>170 \mathrm{mg} / \mathrm{dl}$ in preterm babies (or $>150 \mathrm{mg} / \mathrm{dl}$ among term babies) and glucose $<50 \%$ of serum glucose, determined just prior to lumbar tap.

The Interpretation of neonatal CSF parameters is also difficult when the fluid is blood stained, as this obviously changes both the cellular and biochemical constituents of the fluid. One of the traditional methods of resolving the debacle is to count both the erythrocytes and leucocytes in the fluid and use the physiological ratio of 700 erythrocytes to one leucocyte as the cut-off point from normal. In a more recent US study, it was reported amongst others that, in a cohort of newborn infants, CSF protein increased by $2 \mathrm{mg} / \mathrm{dl}$ for every 1000 erythrocytes in the CSF even in spite of the presence of pleocytosis. ${ }^{31}$ This method may not require sophisticated kits or personnel and thus, can be studied further for its clinical applicability in the developing world.

The CSF gets sterilized rapidly following exposure to antibiotics. It takes just about two hours for meningococcus, and up to six hours for pneumococcus. ${ }^{32}$ This is important in the situations of pre-hospital care antibiotic use which is common in parts of the world where antibiotic use is poorly regulated. The CSF culture findings may be altered by prior antibiotic use but antibiotics rarely interfere with CSF protein or glucose. In this situation, bacterial antigen detection serological methods would be most useful. Even in the absence of prior exposure to antibiotics, delay in processing CSF for up to four hours, has been reported to cause progressive decline in CSF glucose and white cell counts. ${ }^{33}$ This is very relevant to the practice in the developing world where inadequate laboratory services (in terms of personnel, infrastructures and equipment) may warrant delays in processing CSF samples. Therefore, practitioners in this part of the world need to take these variables into consideration when interpreting CSF parameters.

To a large extent, the use of molecular and serologic methods such as the polymerase chain reaction (PCR), which does not require the presence of live organisms for diagnosis, have improved the diagnosis of meningitis on the CSF. Commercial kits for performing the sero- 
logic tests for Streptococcus pneumoniae, Group B Streptococcus, E. coli and Haemophilus influenzae are available in most laboratories in the developed world but not yet in the resource-poor parts of the developing world. PCR has been shown to detect $100 \%$ cases of bacterial meningitis in a cohort of Greek children while culture detected only $21.4 \% .{ }^{34}$ In addition, PCR, may, in the future be used to prognosticate since a study of quantitative PCR on blood, showed a correlation between disease severity and meningococcal bacterial DNA load among older children. ${ }^{35}$ Although, meningococcus is not a common cause of newborn meningitis. However, it is very likely that a similar principle may apply to other pathogens with respect to PCR and severity of illness.

\section{Neuro-imaging}

Neuro-imaging is required for the diagnosis of intracerebral collections, structural focal lesions and ventricular dilatation. These features may affect response to antibiotic treatment of meningitis. Therefore, poor clinical response despite adequate therapy is an indication for neuro-imaging in newborn meningitis. The most basic of such imaging method in use in the developing world include trans-fontanelle ultrasonography and computerized tomographic (CT) scans of the brain. Unfortunately, the wide variation in the size of normal lateral ventricles make CT scans less reliable in diagnosing truly dilated ventricles in the newborn. ${ }^{36}$ More efficient imaging facilities like the Magnetic Resonance Imaging are unfortunately, not available for routine use in most centres in this part of the world.

\section{Other investigations}

Full blood count, C-reactive protein (CRP), clotting studies, and urea and electrolytes are useful ancillary tests in sepsis work-up. Leucopaenia and elevated CRP are known to be more consistent with the diagnosis of severe bacterial infection in the newborn. ${ }^{37}$ Elevated procalcitonin (with sensitivity of $90 \%$ and specificity of $65 \%$ ) was recently shown, in a systematic review, to have good diagnostic accuracy especially for late-onset sepsis where meningitis occur commonly. ${ }^{38}$

\section{Treatment}

The three major aspects of treatment of bacterial meningitis include (1) antibiotic therapy (2) fluid restriction (3) adjunctive therapy. Central to the understanding of the relevance of these major aspects is the basic pathology and pathogenesis of bacterial meningitis. Following the penetration of pathogens into the subarachnoid space, the destruction of the bacterial cell walls in the meninges may occur spontaneously or following antibiotic treatment. The destruction of the bacterial cell walls results in the release of antigenic components such as peptidoglycan and teichoic acid into the subarachnoid space. The released bacterial toxins and cell wall components provoke the release of cytokines such as interleukins (IL-1 $\beta$ ), tumour necrosis factor- $\gamma$ (TNF) and platelets aggregation factor (PAF). These agents in turn, offset an inflammatory cascade characterized by increased vascular permeability, polymorphonuclear migration and activities and production of exudates and cellular debris. Ultimately, these inflammatory events result in cerebral oedema, elevated intracranial pressure, reduced cerebral perfusion, cerebritis, neuritis and vasculitis. ${ }^{39}$ The end-result of all these pathologic features include ischaemia, infarction and atrophy of neural tissues. Short and long-term morbidities occur in infants with bacterial meningitis as a result of the aforementioned pathologic changes. Some of the acute morbidities in meningitis include cerebral oedema, subdural effusion, subdural empyema, venous sinus thrombosis, cranial nerve palsies and hydrocephalus. Long term neurologic deficits in meningitis include hearing loss, cortical blindness, strabismus, speech disorders, behavioural disorders, motor deficits particularly hemiplegia, mental retardation and seizure disorders. ${ }^{5,9}$

\section{Antibiotic treatment}

The goal of antibiotic treatment in meningitis is rapid sterilization of the CSF. This explains why antibiotic therapy is highly recommended when meningitis is clinically suspected even when definite investigations are not feasible or delayed. In standard practice where microbiological diagnosis could be reliably made, the choice of antibiotic depends on the organism isolated. However, in most cases especially in most parts of the developing world, the initial treatment is usually empirical pending the availability of sensitivity reports from the laboratory. This empirical treatment depends on the known epidemiology of the likely organisms as well as the local antibiotic resistance patterns. When the bloodbrain barrier is inflamed as it occurs in meningitis, the permeability is increased and the penetration of most antibiotics into the CSF is improved. It is important that, the antibiotic chosen for empirical treatment should have good penetration into the CSF and achieve adequate minimum bactericidal concentration (MBC) for the organism. ${ }^{40}$ These properties are important for rapid sterilization of the CSF which impacts on the survival of the affected infants.

The traditional treatment of neonatal meningitis involves ampicillin and gentamicin. Although, the penicillins generally have poor CSF penetration when the meninges are inflamed, ${ }^{41}$ adequate concentrations can be delivered into the CSF with more frequent and higher doses.

On the other hand, gentamicin readily penetrates the inflamed blood-brain-barrier but rarely achieves the minimum bactericidal concentration for the pathogens. Increasing the therapeutic dose of gentamicin in order to achieve higher minimum bactericidal concentration in the CSF may be harmful since the drug ordinarily has a narrow therapeutic index and can readily cause ototoxicity and nephrotoxicity. ${ }^{41}$ This is particularly challenging in the resource-constrained parts of the world where facilities for monitoring serum drug levels are not available. In an attempt to circumvent this challenge, trials of intraventricular administration of antibiotics in meningi- 
tis were conducted but the findings were disappointing with observed increased mortality. ${ }^{42,43}$

The WHO recommended initial antibiotic combination of a penicillin (e.g. ampicillin or penicillin G) and an aminoglycoside (e.g. gentamicin) or a third-generation cephalosporin (e.g. ceftriaxone or cefotaxime) for the treatment of meningitis in young infants aged < 60days. ${ }^{44}$ A recent systematic review ${ }^{45}$ which compared the use of third generations and the traditional antibiotics (ampicillin, penicillin and chloramphenicol in various combinations), found no significant difference in terms of death, deafness and treatment failures among subjects with meningitis. However, the review covered all studies of bacterial meningitis irrespective of age hence the difficulty in generalizing the results of the review.

In the developed world, the first line therapy is usually ampicillin with gentamicin or ampicillin with cefotaxime or ceftriaxone although the latter has the tendency to cause displace bilirubin from albumin binding sites. ${ }^{37}$ The third generation cephalosporins are effective on a wide range of pathogens causing meningitis, including the aminoglycoside-resistant strains. Unfortunately, this class of antibiotics has not been shown to be particularly effective on Listeria monocytogenes, thus, they are not recommended for monotherapy in places where Listeriosis is common. Nevertheless, they have good blood-brain-barrier penetration and achieve adequate minimum bactericidal concentrations for most organisms in the CSF. ${ }^{46}$ Ampicillin is effective on GBS, the coliforms and Listeria monocytogenes. Although, Listeria monocytogenes and GBS are reportedly uncommon in most parts of the developing world, most physicians also adopt the combination of ampicillin and cefotaxime in the empirical treatment of meningitis in the newborn. The third-generation cephalosporins (cefotaxime and ceftriaxone) are active against the major pathogens of neonates worldwide, including aminoglycoside-resistant strains. A recent Nigerian study reported remarkable sensitivity of pathogens in newborn septicaemia to ceftriaxone, cefotaxime and ceftazidime. ${ }^{23}$ Thus, these drugs can also be reliably used in the empirical treatment of meningitis.

\section{Antimicrobial resistance}

Antibiotic resistance is a major challenge confronting practitioners worldwide. ${ }^{25}$ This, to a large extent, makes global recommendations on the choice of drugs which are useful for the empirical treatment of severe infections in the newborn difficult. A recent study of newborn septicaemia in Sagamu, Nigeria reported overall resistance of $70.3 \%$ to ampicillin and $43.6 \%$ to gentamicin. ${ }^{23}$ Specifically, the resistance of Staphylococccus aureus to ampicillin and gentamicin were $66.7 \%$ and $37.5 \%$ respectively in the report from Sagamu.

This observation is important in view of the predominance of Staphylococcus aureus as a leading pathogen in newborn meningitis as reported in another Nigerian study. ${ }^{5}$ In addition, the Gram negative bacilli have been shown to have high resistance to ampicillin and gentamicin. ${ }^{17,19}$ These reports have significant implications for the principle of using ampicillin and gentamicin for the empirical treatment of newborn meningitis in this part of the world.

\section{Duration of treatment and choice of antibiotic}

Antibiotic therapy often requires modification once antibiotic susceptibility testing becomes available. The antibiotic treatment of meningitis in the newborn is traditionally prolonged because of the challenges of penetration of CSF and achievement of minimum bactericidal concentration earlier mentioned. At present, there is no statistical evidence to specifically guide the duration of antibiotic treatment in neonatal meningitis. However, the general principle is that the duration of antibiotic treatment depends on the organism isolated. For Gram negative bacilli, parenteral administration of antibiotics should be continued for a minimum of three weeks or for 14 days after the sterilization of the CSF. ${ }^{37}$ This prolongation of therapy is important because of delayed sterilization of the CSF in Gram-negative bacilli meningitis. The latter fact may explain the high mortality and poorer outcome associated with Gram negative bacilli meningitis. For GBS, the minimum duration of treatment is 14 days while Staphylococcus aureus meningitis should be treated for up to three weeks in order to reduce the risk of cerebral abscess formation.

It is recommended that LP should be repeated 24 to 48 hours after the commencement of therapy to ascertain sterilization of the CSF. However, with cefotaxime and ceftriaxone, an Australian study of childhood meningitis demonstrated that the lowest CSF concentrations of both drugs were several times higher than the minimum bactericidal concentrations for the organisms (meningococcus, pneumococcus and Haemophilus influenzae). ${ }^{47}$ On the basis of these findings, the authors recommended that repeat LP was not necessary when treating meningitis due to these organisms with either of these drugs. Thus, it may be attractive to recommend either of these drugs for the empirical treatment of newborn meningitis in places where significant resistance to the drugs have not been reported and avoid repeat LP.

In some unusual cases, inflammatory changes in the CSF may persist despite adequate antibiotic treatment as a result of obstructive ventriculitis, subdural empyema, cerebral abscess or intracranial vessel thrombi. Persistent CSF inflammation is an indication for neuroimaging and review of antibiotic therapy. From the results of neuroimaging, other therapeutic measures like surgical interventions may be required in the care of the meningitic infant. In addition to neuroimaging, antibiotic therapy may need to be prolonged. Subsequent CSF examination is unnecessary if the CSF has been sterilized by 48 hours of therapy and the clinical course has been satisfactory.

Repeat LP at the end of therapy, hitherto recommended, is currently unpopular in the UK. ${ }^{48}$ A study of a small 
number of babies also showed that a repeat LP was unnecessary if clinical response to treatment was satisfactory. This suggestion was based on the fact that neither normal CSF at the end of treatment nor abnormal CSF findings accurately predicted cure or relapse. ${ }^{49}$ Therefore, practitioners need to weight the benefit of a repeat LP at the end of treatment against the attendant risks.

Trials of shorter duration of treatment are on-going among older children. A randomized trial among 100 infants with meningitis showed that four days of ceftriaxone treatment is as effective as seven days with no difference in complications and treatment failures. ${ }^{50} \mathrm{~A}$ more recent double-blind randomized trial in Malawi, showed no significant difference in relapse or treatment failure rate among children with meningitis treated with antibiotics for five days or 10 days. ${ }^{51}$ This study suggested that discontinuation of antibiotics after the fifth day of ceftriaxone among children of post-neonatal age who remained stable was safe. Similar trials in the newborn period are highly desired.

Similarly, a randomized controlled trial of seven-day versus 14-day antibiotic treatment for neonatal sepsis showed similar treatment failure rates in both groups prompting a recommendation of shorter duration of treatment. ${ }^{52}$ For clinical usefulness, large scale controlled trials are also required. It will be interesting to know if these findings may be safely extrapolated to other invasive newborn diseases like meningitis.

\section{Use of dexamethasone}

Bacterial meningitis is characterized by high mortality and severe neurologic sequelae among most survivors. It is believed that most of the sequelae are as a result of the damage to neural tissue during the acute inflammatory process that characterizes bacterial meningitis. Therefore, the use of corticosteroids as adjuncts in the treatment of bacterial meningitis is intended to attenuate the acute inflammatory process, minimize tissue damage and ultimately improve clinical outcomes both in the short and long term.

The timing of administration of corticosteroids in meningitis is also important in the determination of its efficacy. In childhood bacterial meningitis, the administration of intravenous dexamethasone before or along with the first dose of antibiotics has been reported to be more beneficial compared to administration after the commencement of antibiotics. ${ }^{53}$ It is standard practice to administer dexamethasone in divided doses for four days. There is no evidence that the drug is more effective when given for longer or shorter periods but the risk of adverse effects appears to increase with the duration of administration. ${ }^{54}$

Although, the use of adjuvant corticosteroids has been traditionally employed in the treatment of meningitis among children of post-neonatal age and adults, randomized and non-randomized studies have given conflicting reports concerning the effectiveness of adjuvant dexamethasone in improving the survival and reducing neurologic deficits including hearing loss in meningitis among children, particularly in low-income countries. ${ }^{55}$ At present, the best evidence for the benefits of dexamethasone is in $\mathrm{H}$ influenzae type $\mathrm{b}$ meningitis where better audiologic outcome has been clearly demonstrated. $^{56}$

Studies of the use of dexamethasone in meningitic newborns are particularly sparse. Therefore, current treatment guidelines for newborn meningitis exclude adjunctive dexamethasone therapy. Nevertheless, there are reports of likely usefulness of dexamethasone in the reduction of overall mortality as well as reduction in neurologic sequelae among survivors of newborn meningitis. A non-randomized study of Australian infants between 1953 and 1961 reported mortality rate of $41 \%$ in the steroid-treated group compared with $75 \%$ of nontreated group. Despite better survival, there was no impressive difference in the occurrence of neurologic sequelae in both groups. ${ }^{57}$ Another non-randomized study of newborns with bacterial meningitis in Nigeria between 1992 and 1995 revealed lower mortality and higher frequency of full recovery among babies treated with adjuvant dexamethasone. ${ }^{5}$ These two studies, despite their demonstration of usefulness of dexamethasone therapy in the newborn, could not be used as a universal guide for treatment because they were not randomized controlled trials. Unfortunately, the most frequently cited study which questioned the usefulness of dexamethasone was randomized and controlled. ${ }^{58}$ The latter study of babies with meningitis in Jordan showed similar case fatality rates (22\% Vs $28 \%$ ) and neurologic sequelae (30\% Vs 39\%) among babies treated with or without dexamethasone.

Several other studies among older infants and children have demonstrated conflicting reports about the efficacy of adjunct dexamethasone therapy in meningitis. ${ }^{53,59}$ The findings from these studies could not be scientifically extrapolated to newborns because subgroup analysis was not carried out for children aged 6 weeks to 12 weeks. The extrapolation could have been useful given the understanding that the immune characteristics and range of pathogens causing serious infections at that age (6 weeks to 12 weeks) are usually similar to those of the newborn period. Nevertheless, till date, there is no clear high-power statistical evidence that the use of corticosteroids in neonatal meningitis truly improves the outcome and prevents neurologic complications of the disease. Neither is there any clear high-power statistical evidence that the use of corticosteroids in neonatal meningitis truly lacks benefits in terms of the outcome of the disease. Systematic reviews of the available controlled trials are desired.

\section{Use of intravenous fluids}

Other important supportive care in the management of meningitis includes anticonvulsant therapy, use of inotropes and fluid management. Unfortunately, there are no controlled clinical trials with respect to the use of 
these measures among newborns. In general, it is a common practice to restrict fluids to two thirds or three quarters of the daily maintenance during the management of childhood meningitis. The basis for this practice is the need to reduce the likelihood of the syndrome of inappropriate secretion of antidiuretic hormone (SIADH). SIADH is characterized by hyponatraemia, fluid retention and a tendency to worsen cerebral oedema in meningitis. Therefore, practitioners reduce fluid therapy in children with meningitis in the hope of preventing SIADH.

The true benefits of this practice are yet to be documented. In the first place, the pathogenesis of SIADH in meningitis remains unclear and the reported incidence of SIADH in meningitis varies considerably. These facts cast a lot of doubt on the usefulness of the practice of fluid restriction in this instance.

In addition, a significant proportion of children with meningitis particularly after delay in presentation presents with dehydration or hypovolaemia and are in dire need of fluid resuscitation. ${ }^{60}$ It will be dangerous to rigidly restrict fluid therapy in such situation.

Nevertheless, the clinical dilemma of whether fluids should be restricted or not continues to generate disparities in the pattern of clinical practice as well as difficulties in interpreting mortality figures in childhood meningitis.

Current thinking suggests that the increased extracellular fluid, the appropriate increased secretion of $\mathrm{ADH}$, and mild systemic hypertension occurring in situations of raised intracranial pressure are compensatory mechanisms. These physiologic changes are required to overcome the raised intracranial pressure and to maintain adequate cerebral blood flow and perfusion. Therefore, fluid restriction is likely to reduce the efficacy of the compensatory mechanisms and thus, increase the likelihood of adverse outcome. ${ }^{61} \mathrm{~A}$ systematic reviews of controlled trials among children showed no significant difference in number of deaths and acute severe neurological sequelae among maintenance-fluid and restricted -fluid groups. ${ }^{62}$ Further subgroup analyses in the same review showed statistically significant difference in favour of the maintenance-fluid group in terms of spasticity, seizures at 72 hours and 14 days and chronic severe neurological sequelae at three-months follow up.

The current body of scientific evidence no longer supports the practice of fluid restriction in the management of childhood meningitis, as there are no benefits either immediately or on long term basis. Rather, it is more attractive to carefully assess infants with meningitis for possible dehydration, correct dehydration appropriately, ensure adequate fluid intake but prevent overhydration.

\section{Treatment of raised intracranial pressure}

Raised intracranial pressure (ICP) is a well recognized complication of meningitis among older children but this is not a cause for concern among newborns with meningitis. Measures aimed at reducing intracranial pressure are only used among older children who are more at risk of significantly raised intracranial pressure as a result of non-expansible cranium. Osmotic agents shift fluids from the extravascular to the intravascular space, resulting in a reduction of intracranial pressure. A recent study among children aged 2 months to 12 years suggested that glycerol-induced increment in osmolality and reduction in CSF volume may be mechanism by which glycerol reduces intra-cranial pressure. ${ }^{63}$ Initial placebo-controlled trials of glycerol with or without dexamethasone in childhood meningitis have demonstrated overall better neurologic sequelae among the cases but not with respect to deafness. ${ }^{64}$ More trials of other osmotic diuretics such as $20 \%$ mannitol, glycerol and hypertonic saline in the treatment of raised ICP are still on-going in different parts of the world. It may seem, by virtue of current knowledge, that meningitic neonates do not desperately require osmotic therapy. However, controlled trials are still needed to prove this assumption.

\section{Prevention}

Neonatal meningitis is characterized by high mortality and severe morbidities which cause handicaps. A study of 111 children aged between 9 and 10 years who survived neonatal meningitis in England and Wales between 1985 and 1989 showed worse general outcome compared to non-meningitic matched controls. The survivors of neonatal meningitis had less mean intelligence quotient, higher frequency of motor impairment, seizures, hydrocephalus and hearing loss. ${ }^{9}$ The spectrum of neurologic sequelae reported in the UK study was not different from the pattern reported from Nigeria. ${ }^{5}$

Given the background of generally poor outcome in neonatal meningitis, prevention is highly desired. The preventive measures in newborn meningitis cannot be separated from those of newborn sepsis generally. These may include routine screening of pregnant women for urinary tract infection, early intervention following spontaneous rupture of fetal membranes and hygienic birth generally. For the developed world and other places where GBS is reportedly predominant, prenatal screening for GBS, appropriate treatment and intrapartum antibiotic prophylaxis are useful practices. ${ }^{65}$ Instructively, these practices only reduce the burden of earlyonset neonatal disease whereas GBS meningitis commonly presents as late-onset sepsis.

Early recognition and prompt commencement of appropriate antibiotic therapy are also desired to minimize the risk of poor outcome in neonatal meningitis. This constitutes major challenge in most parts of the developing world where most ill babies present late to the hospitals. The advent of vaccines against Haemophilus influenzae and Streptococcus pneumoniae infections might have changed the epidemiology of childhood meningitis in the developed world but these have not impacted on the newborn period where the vaccines are not routinely indicated. 


\section{Conclusion}

While efforts are on-going to reduce the incidence of newborn meningitis in the resource-poor parts of the toworld, management strategies also need to be improved enhance survival and reduce neurologic sequelae among the survivors. To achieve this, more research is coveted particularly with respect to optimal antibiotic therapy as earlier suggested. ${ }^{66}$ Specifically, large scale, multi-centred controlled trials are desired to provide evidences for guidance with respect to fluid management and use of steroids and osmotic agents in neonatal meningitis.

Conflict of interest: None

Funding: None

\section{References}

1. World Health Organization. Antimicrobial and support therapy for bacterial meningitis in children. Report of the meeting of 18-20 June 1997, Geneva Switzerland. WHO/CHD/98.6; WHO/EMC/ BAC/98.2. 1998

2. Delouvois J, Blackbourn J, Hurley $\mathrm{R}$ et al. Infantile meningitis in England and Wales: a two year study. Arch Dis Child 1991;66: 603-607.

3. Holt D, Haket S, Louvouis JD et al. Neonatal meningitis in England and wales: ten years on. Arch Dis Child Fetal and Neonatal Ed 2001;84: F85-F89.

4. Airede AI. Neonatal bacterial meningitis in the middle belt of Nigeria. Dev Med Child Neurol 1993;35:424-430.

5. Airede KI, Adeyemi O, Ibrahim T. Neonatal bacterial meningitis and dexamethasone adjunctive usage in Nigeria. Niger J Clin Pract 2008;11:235-245.

6. Moreno MT, vargas S, Poveda R, Saez-liorens X. Neonatal sepsis and meningitis in a developing Latin American country. Pediatr Infect Dis J 1994;13:516-520.

7. Stoll B. The global impact of neonatal infection. Clin Perinatol 1997;24:1-21.

8. Harvey D, Holt D, Bedford H. Bacterial meningitis in the newborn: a prospective study of mortality and morbidity. Semin Perinatol 1999;23:218-225.

9. Stevens JP, Eames M, Kent A, Halket S, Holt D, Harvey D. Long term outcome of neonatal meningitis. Arch Dis Child Fetal Neonatal Ed 2003;88:F179-F184.

10. Ambe JP, Gasi IS, Mava Y. Review of neonatal infections in University of Maiduguri Teaching Hospital: common bacterial pathogens seen. Niger J Clin Pract 2007;10:290-293.
11. Chowdhury HR, Thompson S, Ali Mohammed, Alam N, Yunus M, Streatfield PK. Causes of neonatal deaths in a rural subdistrict of Bangladesh: Implications for intervention. Hlth Popul Nutr 2010;28:375-382.

12. Weber MW, Carlin JB, Gatchalian $\mathrm{S}$ et al. Predictors of neonatal sepsis in developing countries. Pediatr Infect Dis J 2003;22:711-716.

13. Best J, Hughes S. Evidence behind the WHO Guidelines: hospital care for children-what are the useful clinical features of bacterial meningitis found in infants and children? J Trop Pediatr 2008;54:8386.

14. Chien HC, Chiu N, Li W, Huang F. Characteristics of neonatal bacterial meningitis in a teaching hospital in Taiwan from 1984-1997. $J$ Microbiol Immunol Infect 2000;33:100-104.

15. Gaschignard J, Levy C, Romain O et al. Neonatal bacterial meningitis: 444 Cases in 7 years. Pediatr Infect Dis J 2000;30:212 - 217.

16. Longe C, Omene J, Okolo A. Neonatal meningitis in Nigerian infants. Acta Paediatr Scand 1984;73:477-481.

17. Laving AMR, Musoke RN, Wasunna AO, Revathi G. Neonatal bacterial meningitis at the newborn unit of Kenyatta national hospital. East Afr Med J 2003;80:456-462.

18. Nathoo K, Pazvakavamba I, Chidede $\mathrm{O}$ et al. Neonatal meningitis in Harare, Zimbabwe: a 2-year review. Ann Trop Paediatr 1991;11:11-15.

19. Chang CJ, Chang WN, Huang LT et al. Neonatal bacterial meningitis in southern Taiwan. Pediatr Neurol 2003;29: 288 - 294.

20. Adejuyigbe OE, Adeodu OO, Ako -Nai KA, Taiwo O, Owa JA. Septicaemia in high-risk neonates at a teaching hospital in Ile-Ife, Nigeria. East Afr Med J 2001;78:540543.
21. Mokuolu AO, Jiya N, Adesiyan OO. Neonatal septicaemia in Ilorin: bacterial pathogens and antibiotic sensitivity pattern. Afr $J$ Med Med Sci 2002;31:127-130.

22. Ojukwu JU, Abonyi LE, Ugwu J, Orji IK. Neonatal septicaemia in high risk babies in South-Eastern Nigeira. $J$ Perinat Med 2006;34:166-172.

23. Ogunlesi TA, Ogunfowora OB, Osinupebi OA, Olanrewaju DM. Changing trends in newborn sepsis in Sagamu, Nigeria: Bacterial etiology, risk factors and antibiotic susceptibility. J Paediatr Child Health 2011;47:5-11.

24. Adeboye MA, Obasa TO, Fadeyi A, Adesiyun OO, Mokuolu OA. Haemophilus meningitis in an African neonate: time for active surveillance and institution of appropriate control measure. West Afr J Med 2010;29:275-277.

25. Osrin D, Vergnano S, Costello A. Serious bacterial infections in newborn infants in developing countries. Current Opinion Infect Dis 2004;17:217-224.

26. Garges HP, Moody A, Cotten M et al. Neonatal Meningitis: what is the correlation among cerebrospinal fluid cultures, blood cultures, and cerebrospinal fluid parameters? Pediatr 2006; 117: 10941100.

27. Ajayi OA, Mokuolu OA. Evaluation of neonates with risk for infection/suspected sepsis: Is routine lumbar puncture necessary in the first 72 hours of life? Trop Med Int Health 1997;2:284-8.

28. Weisman LE, Merenstein GB, Steenbarger JR. The effect of lumbar puncture position in sick neonates. Am J Dis Child 1983;137:1077-9.

29. Kestenbaum LA, Ebberson J, Zorc JJ, Hodinka RL, Shah SS. Defining cerebrospinal fluid white blood count reference values in neonates and young infants. Pediatr 2010;125:257264. 
30. Smith PB, Garges HF, Cotton CM, Walsh TJ, Clark RH, Benjamin DK Jr. Meningitis in preterm neonates: importance of cerebrospinal fluid parameters. Am J Perinat 2008;25:421-426.

31. Hines EM. Nigrovic LE, Neuman MI, Shah SS. Adjustment of cerebrospinal fluid protein for red blood cells in neonates and young infants. J Hosp Med 2012;7:325328.

32. Kanegaye JT, Soliemanzadeh P, Bradley JS. Lumbar puncture in pediatric bacterial meningitis: defining the time interval for recovery of cerebrospinal fluid pathogens after parenteral antibiotic pretreatment. Pediatr 2001;108:1169-74.

33. Rajesh NT, Dutta S, Prasad R, Narang A. Effect of delay on neonatal cerebrospinal fluid parameters. Arch Dis Child Fetal Neoonatal Ed 2010;95:F25-F29.

34. Papavasileiou K, Papavasileiou E, Tzanakaki G, Voyatzi A, Kremastinou J, Chatzipanagiotou S. Acute bacterial meningitis cases diagnosed by culture and PCR in a children's hospital throughout a 9year period (2000-2008) in Athens, Greece. Mol Diagn Ther 2011;15:109-113.

35. Hackett SJ, Guiver M, Marsh J, et al. Meningococcal bacterial DNA load at presentation correlates with disease severity. Arch Dis Child 2002;86:44-6.

36. Haslam RH. Role of computed tomography in the early management of bacterial meningitis. $J$ Pediatr 1991;119:157-9.

37. Stephenson T, Marlow N, Watkin S, Grant J (eds). Fetal and neonatal infection. In: Pocket Neonatology. 1st ed. Edinburgh: Churchill Livingstone, 2008:241-69.

38. Vouloumanou EK, Plesssa E, karageorgopoulos DE, Mantadakis E, Falagas ME. Serum procalcitonin as a diagnostic marker for neonatal sepsis: a systematic review and meta-analysis. Intensive Care Med 2011;37:747-762.

39. Mustafa MM, Ramilo O, Saez Liorens X, Olsen KD, Magness RR, McCracken GH Jnr. Cerebrospinal fluid prostaglandins, interleukins- 1 beta and tumor necrosis factor in bacterial meningitis: clinical and laboratory correlations in placebo treated and dexamethasone treated patients. Am J Dis Child 1990;144: 883 - 887.

40. Tauber MG, Sande MA. General principles of therapy of pyogenic meningitis. Infect Dis Clin North Am 1990;4:661-76.
41. Rang H, Dale M, Ritter J, Moore P. Pharmacology, 5th edn. Churchill Livingstone, London.2003.

42. McCracken GH, Mize SG, Threlkeld N. Intraventricular gentamicin therapy in gram-negative bacillary meningitis of infancy. Report of the Second Neonatal Meningitis Cooperative Study group. Lancet 1980;1:787-791.

43. Shah SS, Ohlsson A, Shah VS. Intraventricular antibiotics for bacterial meningitis in neonates. Cochrane Database of Systematic Reviews 2008 Issue 4, Art No.: CD004496

44. WHO. Pocket Book of Hospital Care for Children - Guidelines for the Management of Common Illnesses with Limited Resources. World Health Organisation, Geneva. 2005.

45. Prasad K, Kumar A, Singhal T, gupta PK. Third-generation cephalosporins versus conventional antibiotics for treating acute bacterial meningitis. Cochrane Database of Systematic Reviews 2007 Issue 4, Art No.: CD001832.

46. Cherubin CE, Eng RHK, Norrby R, Modai J, Humbert G, Overturf G. Penetration of newer cephalosporins into cerebrospinal fluid. Rev Infect Dis 1989;11: 526-546.

47. Goldwater PN. Cefotaxime and ceftriaxone cerebrospinal fluid levels during treatment of bacterial meningitis in children. Int J Antimicrob Agents 2005;26:408-11.

48. Agarwal R, Emmerson AJ. Should repeat lumbar punctures be routinely done in neonates with bacterial meningitis? Results of a survey into clinical practice. Arch Dis Child 2001;84:451-2.

49. Schaad UB, Nelson JD, McCracken GH Jr. Recrudescence and relapse in bacterial meningitis of childhood. Pediatr 1981;67:188 -95 .

50. Roine I, Ledermann W, Foncea LM, et al. Randomised trial of four vs seven days of ceftriaxone treatment for bacterial meningitis in children with rapid initial recovery. Pediatr Infect Dis $J$ 2000;19:219-22.

51. Molyneux E, Nizami SQ, Saha S et al. 5 versus 10 days of treatment with ceftriaxone for bacterial meningitis in children: a double-blind randomized equivalence study. Lancet 2011;377:1837-1845.

52. Chowdhary G, Dutta S, Narrang A. Randomized controlled trial of 7-day vs 14-day antibiotics for neonatal sepsis. J Trop Pediatr 2006;52:427-432.
53. Odio CM, Faingezight I, Paris M et al. The beneficial effects of early dexamethasone administration in infants and children with bacterial meningitis. New Eng J Med 1991;324:1525 - 1531.

54. McIntyre PB, Berkey CS, King $\mathrm{SM}$ et al. Dexamethasone as adjunctive therapy in bacterial meningitis. A meta-analysis of randomized clinical trials since 1988 . JAMA 1997;278:925 - 931.

55. Van de Beek D, de Gans J, McIntyre P, Prasad K. Corticosteroids for acute bacterial meningitis. Cochrane Database of Systematic Reviews 2007 24(1): CD004405.

56. Schaad UB, Lips U, Gnehm HE, et al. Dexamethasone therapy for bacterial meningitis in children. Lancet 1993;342:457-61.

57. Yu JS, Grauaug A. Purulent meningitis in the neonatal period. Arch Dis Child 1963;38: 391 - 396

58. Daoud AS, Batieha A, Al-Sheyyab M, Abuekteish F, Obeidat A, Mahafza T. Lack of effectiveness of dexamethasone in neonatal bacterial meningitis. Eur J Pediatr 1999;158: $230-233$.

59. Molyneux EM, Walsh AL, Forsyth $\mathrm{H}$ et al. Dexamethasone treatment in childhood bacterial meningitis in Malawi: a randomized controlled trial. Lancet 2002;360: 211 $-218$.

60. Prince AS, Neu HC. Fluid management in Hameophilus influenza meningitis. Infect 1980;8:5-7.

61. Singhi SC, Singhi PD, Srinivas B, et al. Fluid restriction does not improve the outcome of acute meningitis. Pediatr Infect Dis J 1995; 14:495-503.

62. Maconochie IK, Bauner JH, Stewart M. Fluid therapy for acute bacterial meningitis. Cochrane Database of Systematic Reviews 2008, Issue 1 Art No.: CD004786

63. Singhi S, Jarvinen A, Peltola H. Increase in serum osmolality is possible mechanism for the beneficial effects of glycerol in childhood bacterial meningitis. Pediatr Infect Dis J 2008; 27: 892-896.

64. Peltola H, Rome I, Fernandez J et al. Adjuvant glycerol and/or dexamethasone to improve the outcome of childhood bacterial meningitis: a prospective, randomized, doubleblinded, placebo-controlled trial. Clin Infect Dis 2007;45:1277-12686.

65. American Academy of Pediatrics. Revised guidelines for prevention of early-onset Group B Streptococcal infection. Pediatr 1997;99:489-96.

66. Furyk JS, Swann O, Molyneux E. Systematic review: neonatal meningitis in the developing world. Trop Med Int Health 2011;16:672 - 679. 